\title{
A CURVA DE KUZNETS AMBIENTAL PARA A REGIÃO NORTE DO BRASIL ENTRE OS ANOS DE 2002 A 2015
}

\section{THE ENVIRONMENTAL KUZNETS CURVE FOR THE NORTHERN REGION OF BRAZIL BETWEEN 2002 AND 2015}

\author{
Matheus Gama De Almeida \\ Tarcísio da Costa Lobato ${ }^{2}$
}

\begin{abstract}
A Região Norte brasileira tem sido centro de debates socioambientais por concentrar a maior parte da Amazônia Legal e nela estar ocorrendo um grande crescimento de atividades agropecuárias, florestal, mineral, industrial e outras atividades urbanas. Esta ampliação tem gerado vários impactos e um deles é a deterioração do meio ambiente. Neste ensejo, o presente estudo tem o intuito de modelar uma Curva de Kuznets Ambiental para a região Norte do Brasil para o período 2002 a 2015. Para isso, foram coletados dados sobre o PIB per capita - proxy de crescimento econômico - no IBGE e SIDRA. E os dados anuais da emissão de $\mathrm{CO}_{2}$ - proxy da degradação ambiental - para os sete estados da região norte, foram obtidos através do SEEG. Para a análise dos resultados foi utilizado o método de dados em painel. Verifica-se que Rondônia e Acre possuem as maiores variabilidades de emissão de $\mathrm{CO}_{2}$ nos anos estudados e que Roraima, Pará, Amapá e Tocantins mantiveram uma variabilidade pequena entre os anos. Esses resultados também semelhantes aos encontrados no PIB per capita. Sobre o formato da CKA para a região Norte do Brasil, conclui-se que ela se apresenta na forma de um U não invertido, como proposto na teoria.
\end{abstract}

Palavras-chave: Crescimento econômico. Degradação ambiental. Curva de Kuznets Ambiental.

\begin{abstract}
The northern region of Brazil has been a center of debate because it concentrates most of the Legal Amazon and in it is occurring a great growth of agricultural activities, forestry, mineral, industrial and other urban activities. This expansion has generated several impacts and one of them is the deterioration of the environment. In this context, the present study intends to model an Environmental Kuznets Curve for the northern region of Brazil from 2002 to 2015. For this purpose, data were collected on GDP per capita - proxy for economic growth - in IBGE and SIDRA. And the annual data of the $\mathrm{CO}_{2}$ emission - proxy of the environmental degradation - for the seven states of the northern region were obtained through SEEG. For the analysis of the results will be used the panel data method. It is verified that Rondônia and Acre have the greatest variability of $\mathrm{CO}_{2}$ emission in the years studied and that Roraima, Pará, Amapá and Tocantins maintained a small variability between the years. These results are also similar to those found in GDP per capita. On the EKC format for the Northern region of Brazil, it is concluded that it is presented in the form of a non-inverted $U$, as proposed in the theory.
\end{abstract}

Keywords: Economic growth. Ambiental degradation. Environmental Kuznets curve.

JEL: C23, O47, Q56.

\footnotetext{
1 Graduado em Ciências Econômicas. Universidade Federal do Oeste do Pará. E-mail: matheusgama.s@hotmail.com

2 Doutorando em Economia Aplicada - ESALQ/USP. Mestre em Estatística - UFPA. Bacharel em Estatística - UFPA. Licenciado em Matemática - UEPA. Professor Assistente da Universidade Federal do Oeste do Pará, lotado no curso de Ciências Econômicas. E-mail: tarcisiolobato@yahoo.com.br
} 


\section{INTRODUÇÃO}

Os modelos clássicos do crescimento mostram a acumulação do capital e a geração do capital humano como os principais norteadores que conduzem ao desenvolvimento e a um modo de vida semelhante àquele experimentado pelas economias mais desenvolvidas. No entanto, a grande pressão exercida pelas entidades não governamentais, formuladores de política e pesquisadores tem apresentado as barreiras desses modelos, que não levam em consideração as consequências da utilização abundante dos recursos naturais, o que tem provocado um forte debate acerca da sustentabilidade (ROCHA; KHAN; LIMA, 2013).

A degradação ambiental ocasionada pela esfera econômica, mais especificamente pelo seu crescimento, aumenta diariamente. Essa é uma preocupação oriunda do século passado e vem à tona novamente toda vez que o assunto é o meio ambiente. Principalmente nas últimas décadas isso fica ainda mais evidente, devido ao grande número de acordos firmados entre as nações com o objetivo de reduzir a deterioração ambiental.

Destacado por Ikeda (2016), o último grande acordo firmado, aconteceu na $21^{\text {a }}$ Conferência das Partes ${ }^{3}$ (COP21) da Convenção-Quadro das Nações Unidas sobre Alterações Climáticas (CQNUAC), realizada no ano de 2015. Na qual, foi adotado o Acordo de Paris como novo quadro internacional de esforços de combate ao aquecimento global, que é provocado pela elevação das emissões de gases do efeito estufa. Embora sem validade jurídica, o autor considera como inovadora a ideia de que 195 países se comprometeram a tomar medidas contra o cenário de crescente preocupação com as graves consequências que a humanidade terá de enfrentar se a temperatura média global não for mantida $2^{\circ} \mathrm{C}$ abaixo dos níveis pré-industriais.

Segundo o Sistema de Estimativas de Emissões e Remoções de Gases de Efeito Estufa - SEEG - (2018), o setor de Mudanças do Uso da Terra é o mais dinâmico dos setores abarcados nas estimativas de emissões, devido à maneira que se utiliza o solo no Brasil. Além de ter várias regiões de fronteira agrícola e econômica, o país apresenta uma grande alternância entre os tipos de atividade agropecuária, da qual derivam tanto a emissão quanto a remoção ou fixação de gases do efeito estufa. As emissões consideradas mais relevantes são de dióxido de carbono $\left(\mathrm{CO}_{2}\right)$, que resultam da modificação de florestas em áreas agrícolas ou pastagens.

No Brasil, de acordo com Oliveira et al (2011) a maioria dos estudos que são realizados sobre a degradação de florestas se concentram na região Norte do país, pois esta abarca a maior parte da Amazônia Legal e por causa da ameaça que esse desmatamento oferece ao acervo de biodiversidade e a prestação de serviços ambientais para a conservação das condições climáticas no mundo. Até a década de 1980, o desmatamento nessa região chegava a cerca de $300 \mathrm{mil} \mathrm{km}^{2}$, o que equivale a $6 \%$ da área total. Anos mais tarde, em 2007, já chegava a cerca de $732 \mathrm{mil} \mathrm{km}^{2}$ desmatados, o que corresponde a quase $15 \%$ da área total da região (BRASIL, 2008).

Segundo o "Plano Amazônia Sustentável" (2008), essa região é considerada como uma região heterogênea em vários aspectos, pois mostra sua diversidade natural, social e cultural. Além disso, os sistemas de produção são essenciais não só para a sustentabilidade ambiental, mas também para a dinâmica econômica e a inclusão social. Neste sentido, os setores econômicos mais expressivos da Região

\footnotetext{
${ }^{3}$ A Conferência das Partes (COP) é o órgão supremo da Convenção Quadro das Nações Unidas sobre Mudança do Clima (UNFCCC), que reúne anualmente os países Parte em conferências mundiais. Suas decisões, coletivas e consensuais, só podem ser tomadas se forem aceitas unanimemente pelas Partes, sendo soberanas e valendo para todos os países signatários (MMA, 2018).
}

8 Economia \& Região, Londrina (Pr), v.7, n.1, p.7-25, jan./jun. 2019 
são: agropecuário, florestal, mineral, industrial e atividades urbanas. A relação do desmatamento com o crescimento econômico, portanto, torna-se uma questão de interessante complexidade (OLIVEIRA et al, 2011).

De acordo com Instituto Nacional de Pesquisas Espaciais (INPE), a partir de dados do Deter, o desmatamento na Amazônia Legal entre agosto de 2012 e julho de 2013 foi de $2765,64 \mathrm{~km}^{2}$, com uma elevação de $35 \%$ quando comparado com o período entre agosto de 2011 e julho de 2012. Este período estudado pelo INPE é referente aos meses do "calendário do desmatamento", que possui relação com as chuvas e as atividades agrícolas no bioma. Esses dados indicam as proporções que tem tomado o aumento do desmatamento no bioma brasileiro.

$\mathrm{O}$ aumento das emissões de gases do efeito estufa, mais especificamente do $\mathrm{CO}_{2}$, proveniente da atividade econômica dos países é tema de uma discussão iniciada em 1991, quando Grossman e Krueger propuseram em seu artigo que a relação entre a degradação ambiental e o crescimento econômico gera por si só uma qualidade de vida ambiental depois que alcança um determinado ponto. Essa relação ficou conhecida como Curva de Kuznets Ambiental, nome esse derivado do estudo de Simon Kuznets (1955), que chegou a uma curva no formato de $U$ invertido.

A partir de então, vários autores começaram a testar a hipótese da CKA em diversos âmbitos (global, nacional e regional), a fim de confirmar o que sugere a teoria. Para o caso do Brasil, podemos observar uma divisão entre os pesquisadores; alguns autores não conseguiram chegar a uma conclusão sobre formato da curva (LUCENA, 2005), outros conseguiram constatar a existência da CKA como no formato proposto (ROCHA; KHAN; LIMA, 2013), outros chegaram ao formato de U, como Queiroz, Rocha e Queiroz (2014) e a maioria dos autores encontraram uma curva no formato de $\mathrm{N}$ invertido - que representa um movimento cíclico (OLIVEIRA et al, 2011). A nível global o formato encontrado também é de o de N invertido (ARRAES, DINIZ e DINIZ, 2006; CARVALHO, 2008; CARVALHO, 2010; CATALÁN, 2014).

Diante dos elevados índices expostos de degradação ambiental devido ao crescimento econômico, o estudo dessa relação na região Norte se mostra de grande importância, pois além de concentrar a maior parte das florestas tropicais, há um variado leque de atividades econômicas sendo desenvolvido. Sendo assim, faz-se o questionamento se com essas especificidades que a região apresenta chegaremos ao formato da Curva de Kuznets Ambiental proposto pela teoria.

O presente estudo tem o intuito de modelar uma Curva de Kuznets Ambiental para a região Norte do Brasil para o período 2002 a 2015. Além disso, analisar o comportamento da emissão de $\mathrm{CO}_{2}$ e do PIB per capita, propor um modelo econométrico para ajustar a Curva de Kuznets Ambiental a região do estudo e verificar o formato da Curva de Kuznets Ambiental que a região Norte possui.

\section{CURVA DE KUZNETS AMBIENTAL}

A discussão sobre a distribuição de renda e o crescimento econômico de Simon Kuznets (1955) é o maior motivador dos trabalhos que estudam essa relação (SALVATO, 2006). Segundo Bêrni, Marquetti e Kloeckner (2002), esse debate foi iniciado a partir do seu discurso como presidente da Associação Econômica Americana e, posteriormente, em março de 1955 publicou um artigo intitulado "Economic Growth and Income Inequality". Nesse estudo, sua hipótese inicial era de 
que a desigualdade de renda aumenta com o crescimento econômico, atingindo um ápice e, posteriormente, decaí, resultando em uma curva no formato de um $U$ invertido, que ficou conhecida como Curva de Kuznets Original (BARROS; GOMES, 2008). A Figura 1, abaixo, mostra essa relação.

Figura 1 - Curva de Kuznets Original

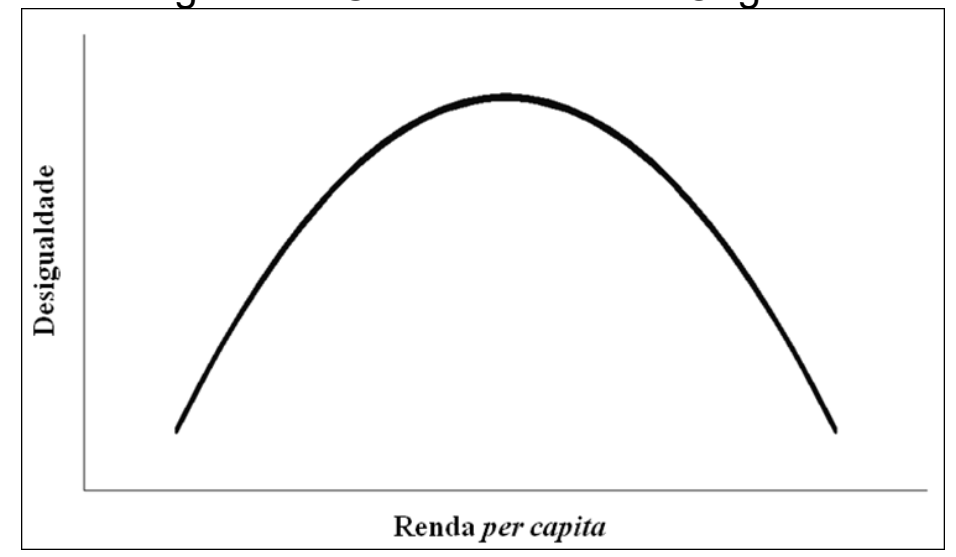

Fonte: Elaborada pelos autores.

Kuznets (1955) já expôs em seu primeiro parágrafo que o tema central daquele artigo era o caráter e as causas no longo prazo das mudanças na distribuição de renda pessoal. Além disso, indagou se a desigualdade na distribuição de renda aumenta ou diminui no decurso de um crescimento econômico do país e quais seriam os fatores que determinam o nível secular e tendências de desigualdades de renda. Ele também expõe a dificuldade em abordar esse campo de estudo por questões de falta de definição e uma escassez incomum de dados.

A partir do estudo seminal de Simon Kuznets em 1955, Grossman e Krueger (1991) relacionaram pela primeira vez o crescimento e conômico com a degradação ambiental, afirmando a existência de uma curva no formato de $U$ invertido, que ficou conhecida como Curva de Kuznets Ambiental (CKA), semelhante à observada na relação entre crescimento econômico e desigualdade de renda proposta por Kuznets.

Ávila e Diniz (2015) afirmam que a CKA demonstra uma relação entre emissões - de material particulado em suspensão (SPM) e dióxido sulfúrico $\left(\mathrm{SO}_{2}\right)$ - e renda no formato de $U$ invertido. Os aludidos autores acrescentam que nas fases iniciais do crescimento econômico, a renda e as emissões possuem uma relação direta, enquanto que quando a renda se eleva e atinge certo nível, as emissões começam a decair. Como se pode observar na Figura 2. 
Figura 2 - Curva de Kuznets Ambiental.

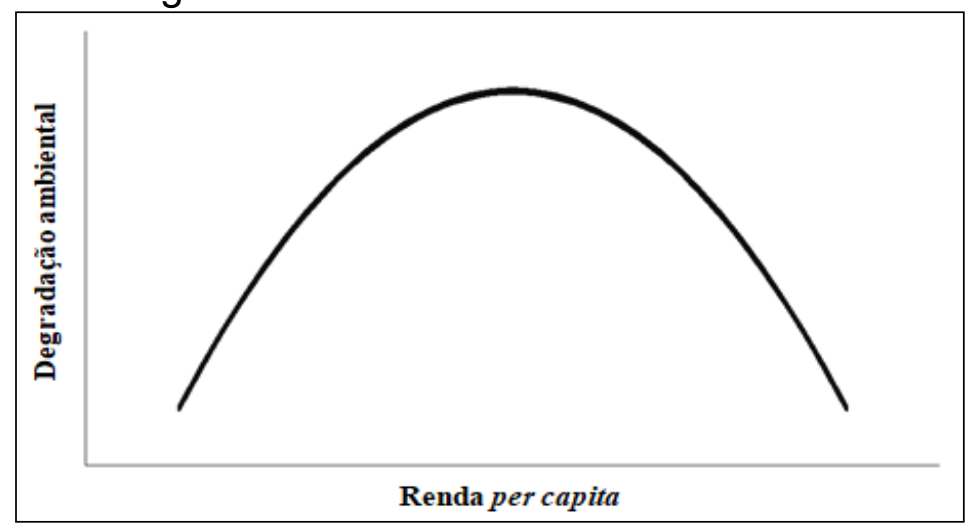

Fonte: Elaborada pelos autores.

Lucena (p. 09, 2005) afirma que a CKA é:

“(...) uma hipótese acerca da relação entre indicadores de degradação ambiental e a renda per capita. Segundo ela, nos estágios iniciais do desenvolvimento econômico, a degradação ambiental e a poluição aumentariam juntos com a renda per capita. Contudo, após certo nível de renda (a partir de agora chamado de "ponto de inflexão"; em inglês, turning point), que varia de acordo com os indicadores estudados, essa tendência se reverteria de tal forma que a qualidade ambiental melhoraria com o crescimento econômico.

De acordo com Carvalho e Almeida (2010), quando um país extremamente pobre obtém um crescimento, a poluição no início cresce pelo fato de que os aumentos na produção geram emissões de poluentes e porque o país, devido a sua pobreza, não tem como uma prioridade o controle da degradação do meio ambiente. A partir do momento que o país adquiriu um grau de afluência, sua prioridade troca para proteção da qualidade ambiental. "Se esse efeito renda é forte o suficiente, causará o declínio da poluição".

Segundo Rocha, Khan e Lima (2013) o estudo de Grossman e Krueger (1991) mostrou as consequências da redução das barreiras comerciais sobre a degradação ambiental, de forma que os impactos da regulação do NAFTA (North American Free Trade Agreement) para os EUA e Canadá consistiriam em uma maior especialização de atividades intensivas em capital humano e físico e na geração de "tecnologias limpas". O benefício líquido em nível global, em síntese, iria resultar em uma massiva movimentação das atividades econômicas "mais sujas" para lugares de produção altamente regulamentados.

Conforme Carvalho e Almeida (p. 589, 2010), "os países passariam por estágios de desenvolvimento, regidos pelas forças de mercado e por mudanças na regulação governamental". Os autores afirmam que em um estudo feito em 1995 por Grossman e Krueger, no estágio inicial, que seria marcado pela mudança de uma economia do campo para uma industrializada, esse crescimento econômico imprimiria uma pressão cada vez maior sobre o meio ambiente, resultante da criação e ampliação do parque industrial. $O$ segundo estágio iria se caracterizar pelo amadurecimento da sociedade e também da infraestrutura industrial. Nesse aspecto, o atendimento das necessidades básicas permite o crescimento de setores menos intensivos em recursos e poluição, e a intensidade de matéria/energia e rejeitos da produção começam a reduzir devido as melhorias técnicas. Por último, no terceiro 
estágio, ocorreria o "descolamento" (de-linking) entre o crescimento econômico e a pressão sobre o meio ambiente, momento em que o crescimento não mais afetaria de forma negativa o meio ambiente.

Consoante Ávila e Diniz (p. 98, 2015), os autores Grossman e Krueger (1995) explicam o formato da curva com base em três efeitos distintos:

\begin{abstract}
"efeito escala, quando um aumento da renda está associado a um aumento das emissões de poluentes para uma dada composição do produto e uma dada tecnologia; efeito composição, quando uma mudança da composição do produto associado a um aumento da renda leva a uma alteração do peso dos produtos menos poluentes no produto total, conduzindo a uma redução das emissões quando o peso dos produtos menos poluentes aumenta; efeito técnica, quando a adoção de tecnologias menos poluentes associada a um aumento da renda leva a uma redução das emissões."
\end{abstract}

Observando a CKA, a relação entre renda e degradação é explicada pelo fato do efeito de escala superar os demais. Esta relação direta acontece nas primeiras fases do crescimento de um país. A relação inversa entre renda e degradação é explicada pela primazia dos efeitos composição e técnica sobre o efeito escala. $\mathrm{O}$ último caso, o efeito técnica, é, possivelmente, o mais relevante. Esta relação inversa ocorre no caso de países mais avançados. O qual pode ocorrer em um nível de renda per capita factível de ser obtido no futuro próximo. Assim sendo, a redução das emissões aconteceria de uma forma natural, sendo desnecessária uma intervenção da autoridade ambiental e a introdução de ineficiências dela decorrentes (ÁVILA; DINIZ, 2015).

Segundo Ávila e Diniz (2015), a hipótese teórica que provém a base da Curva de Kuznets Ambiental é a de que o impacto negativo do efeito escala tende a prevalecer nos estágios iniciais do crescimento econômico, sendo, posteriormente, compensado ao longo do processo de desenvolvimento do país pelos outros dois efeitos, o composição e técnica, os quais tendem a reduzir os níveis da degradação do meio ambiente.

\title{
PROCEDIMENTOS METODOLÓGICOS
}

\section{Área de estudo e coleta dos dados}

O presente estudo analisa a região norte do Brasil. A região compreende os estados do Acre, Amapá, Amazonas, Pará, Rondônia, Roraima e Tocantins. Com uma área de 3.853.676,948 km² é a maior entre as cinco regiões, cobrindo uma área de $45,25 \%$ do território do Brasil. Sua população, também de acordo com o IBGE, era de 17.936.201 milhões de habitantes em 2017. Seu Índice de Desenvolvimento Humano (IDH), de 0.683, é considerado médio e, em comparação com as outras regiões brasileiras, tem o segundo menor IDH, superando apenas o Nordeste.

Além disso, o estudo é realizado do ano de 2002 a 2015. A fim de analisar a relação existente entre o crescimento econômico e degradação ambiental, serão utilizadas proxies para representar essas duas variáveis.

Os dados colhidos para se calcular o PIB per capita, que será utilizado como proxy do crescimento econômico, foram obtidos por meio do IBGE e do Sistema IBGE de Recuperação Automática (SIDRA). No primeiro, foi obtida a população residente 
por estado e, no segundo, foram obtidos os dados do PIB por estado. Esta proxy é comumente usada como um indicador da qualidade de vida. No entanto, não se pode desconsiderar que o PIB pode ter um crescimento, mas os cidadãos de um país podem ficar ainda mais pobres, por exemplo, quando ocorre um desastre ambiental fenômeno atípico - que, por consequência, eleva o PIB, mas não a qualidade de vida. Além disso, o PIB não leva em conta o nível de desigualdade de renda de uma sociedade.

Os dados anuais da emissão de $\mathrm{CO}_{2}$ para os sete estados da região norte, foram obtidos através do SEEG. Será utilizada a emissão de dióxido de carbono como proxy da degradação ambiental, porque esse gás é o que mais influencia no efeito estufa, aumentando assim a temperatura do planeta. De acordo com a ONG World Wide Fund For Nature (WWF), a emissão do $\mathrm{CO}_{2}$ resulta da queima direta de combustíveis fósseis, por exemplo, do carbono, do petróleo e do gás. Os quais são utilizados, principalmente, na produção de energia e transportes.

\section{Instrumental analítico}

Para modelar a Curva de Kuznets Ambiental para a região Norte, no período de 2002 a 2015, ou seja, relacionar o PIB per capita (proxy de crescimento econômico) com a emissão de $\mathrm{CO}_{2}$ (proxy de degradação ambiental), será empregada a técnica de dados em painel. Em seguida, serão feitas as estimações por Mínimos Quadrados Ordinários (MQO) para dados em painel (agrupando observações de séries temporais e de corte transversal), efeito aleatório e efeito fixo. Além dos testes para a escolha do modelo mais adequado, usando a emissão de $\mathrm{CO}_{2}$ como variável dependente e o PIB per capita como independente.

Conforme Hsião (2003), um corte longitudinal, ou painel, é um conjunto de dados, que adota uma dada amostra de indivíduos ao longo do tempo, e, deste modo, oferece uma gama de observações em cada indivíduo na amostra. Dados em painel se tornaram largamente disponíveis nos países já desenvolvidos e também nos que estão em desenvolvimento.

Segundo Baltagi (1995) existem várias vantagens de se usar o método de dados em painel, sendo elas: primeira, a probabilidade de controle da heterogeneidade individual; segunda, um maior poder de informação dos dados, mais variabilidade, menor colinearidade entre as variáveis, maior grau de liberdade e mais eficiência; terceira, uma melhor análise da dinâmica de ajustamento; quarta, a possibilidade de identificar e proporcionar efeitos que os dados de séries temporais ou corte transversal não captam; quinta, a possibilidade de arquitetar e examinar modelos mais complexos; sexto, a possibilidade de melhor cogitar certas variáveis e modelos em nível micro.

Em resumo, de acordo com Gujarati \& Porter (2011) os dados em painel podem deixar a análise aplicada tão rica ao ponto de não ser possível utilizarmos apenas dados de séries temporais ou de corte transversal. No entanto, isso não deve sugerir que não existam problemas com o emprego da modelagem de dados em painel.

\section{Modelo de efeito fixo}

Segundo Fernandes (2016), o modelo de efeito fixo é uma generalização de um modelo constante intercepto inclinação para dados em painel, compreendendo uma variável dummy para os efeitos das variáveis omitidas, que se estão constantes no tempo. Além disso, a autora afirma que o objetivo do efeito fixo é constatar os 
impactos das variáveis independentes variando entre indivíduos e também ao longo do tempo. Para esta finalidade, imagina-se que o intercepto irá variar de um indivíduo para outro, entretanto, é constante ao longo tempo, a fase que os parâmetros são constantes para as entidades e em todos os períodos de tempo.

Gujarati \& Porter (2011) afirmam que o termo "efeitos fixos" deve-se ao fato de que, embora o intercepto possa diferir entre os indivíduos, o intercepto de cada indivíduo não varia com o tempo. De acordo com Costa, Moreira e Loureiro (2015), este modelo é uma regressão no formato clássico $y_{i t}=\alpha_{i t}+\beta^{\prime} x_{i t}+e_{i t}$, onde $E\left[e_{i t}\right]=0$ e $\operatorname{Var}\left[e_{i t}\right]=\sigma^{2}$. E os $x$ são variáveis dummy específicas que correspondem aos mesmos indivíduos quando $j=i$. Logo, o modelo de efeitos fixos pode ser escrito da seguinte maneira:

$$
y_{i y}=\alpha_{i}+\beta_{1} X_{1 i t}+\beta_{2 i t} X_{2 i t}+\ldots \beta_{k} X_{k i t}+e_{i t}
$$

Conforme Wooldridge (2002) no modelo de efeitos fixos o intercepto é específico para cada unidade, podendo estar correlacionado com um ou mais regressores, porém cada intercepto é invariante o tempo, enquanto que no modelo de efeitos aleatórios o intercepto (aleatório) das unidades não possuem correlação com as outras variáveis.

\section{Modelo de efeito aleatório}

No modelo de efeito aleatório, Fernandes (2016) afirma que as variáveis apresentam suposições iguais as do modelo de efeito fixo, assim sendo, o intercepto muda de um indivíduo para outro, mas não no decorrer do tempo, e os parâmetros resposta são constantes para todos os indivíduos e perante todas as etapas de tempo. $\mathrm{O}$ que difere esses dois modelos é o tratamento do intercepto. $\mathrm{O}$ modelo de efeitos aleatórios considera os interceptos como variáveis aleatórias, assim, o modelo entende que os indivíduos possuem dados que são amostras aleatórias de indivíduos com uma população maior. Em função das especificidades desse modelo, o problema de autocorrelação é uma constante, fazendo com que seja necessária a utilização de MQG factíveis (LOUREIRO \& COSTA, 2009).

De acordo com Marques (2000) as vantagens dos modelos de efeitos aleatórios são: a sua capacidade para trabalhar com bases de dados de qualquer dimensão; o fato de a inferência estatística aplicável ser uma mera derivação dos testes de hipóteses usuais; a possibilidade de a maior parte dos problemas e dificuldades poderem ser resolvidos dentro do quadro econométrico tradicional; o fato de ser o modelo de dados em painel estudado com maior profundidade; a facilidade com que são explicados os resultados de estimação, e; e o fato de ser pouco exigente em termos de software econométrico.

Greene (2000) afirma que o modelo de efeitos aleatórios tem a seguinte equação $y_{i t}=\alpha+\beta^{\prime} x_{i t} e_{i t} \mu_{i t}$, o qual é um modelo de regressão generalizado. Todo o termo aleatório tem variância $\operatorname{Var}\left[e_{i}+\mu_{i}\right]=\sigma e_{2}+\sigma \mu_{2}$. O modelo de efeitos aleatórios é dado por:

$$
Y_{i y}=\alpha+\beta_{1} x_{1 i t}+\beta_{2 i t} X_{2 i t}+\ldots+\beta_{k} x_{k i t}+\mu_{i t}
$$

Segundo Gujarati\& Porter (2011) o presente modelo é desenvolvido sob a premissa de que o intercepto é uma extração aleatória de uma população maior, transformando uma unidade de observação para outra. Além disso, é onde os erros originados não são correlacionados com os parâmetros utilizados no modelo. 


\section{Modelo pooled}

Conforme Cameron e Trivedi (2005) apud Reinaldo (2017), o modelo inicial considerado o mais restrito é o modelo para dados agrupados (pooled), pois esse despreza as dimensões (temporal e espacial) dos dados e leva em consideração todos os coeficientes constantes ao longo do tempo e entre os indivíduos, assumindo a forma de dados empilhados. Sendo assim, o método tradicional para estimação dos parâmetros é o método dos mínimos quadrados ordinários e a formulação geral do modelo com $n$ observações em $T$ períodos e $k$ variáveis é dado por:

$$
Y_{i t}=\alpha+x_{0 i t} \beta+\varepsilon_{i t}, i=1, \ldots, n, t=1, \ldots, \mathrm{T}
$$

em que o subscrito $i$ se refere as diferentes unidades de corte transversal e t é o período de tempo que está sendo considerado; $Y_{i t}$ e a variável dependente do indivíduo $i$ no tempo $t ; x_{0 i t}$ um vetor de variáveis explicativas de ordem $1 \times p ; \beta$ um vetor de ordem $p \times 1$ dos coeficientes das variáveis; $\alpha$ o intercepto e $\varepsilon_{i t}$ o termo de erro do $i$-ésimo indivíduo no tempo $t$. Implica-se que os erros $\varepsilon_{i t}$ são independentes e bem disseminados com a média zero e variância $\sigma^{2}$ (REINALDO, 2017).

\section{Testes para escolha do modelo em dados em painel}

Levando em consideração que Baltagi (1995) alertou para o fato de que a utilização de uma especificação imperfeita poderia ocasionar dificuldades de especificação ou inconsistência dos estimadores. Dessa maneira, antes de se diagnosticar a normalidade, homocedasticidade (Teste White) e autocorrelação (Durbin-Watson) é necessário escolher entre os modelos pooled OLS, efeitos fixos e aleatórios. Para isso, irão ser usados os seguintes testes: teste de Chow, o teste de Breusch-Pagan e teste de Hausman.

De acordo com Caldarelli, Camara \& Perdigão (2015), o teste de Chow para dados em painel é um teste do tipo $F$, que considera o modelo restrito de dados empilhados ou pooled como hipótese nula e o modelo de dados em painel de efeitos fixos como hipótese alternativa. Neste caso, com base no teste conjunto das estimativas, a rejeição à hipótese nula aponta que a melhor escolha é o modelo de efeitos fixos. O teste de Breusch-Pagan é um teste do tipo lagrange multiplier, cuja hipótese nula é a $\operatorname{Var}\left(\sigma_{\mu}^{2}\right)=0$ e a alternativa é a $\operatorname{Var}\left(\sigma_{\mu}^{2}\right) \neq 0$. A rejeição da hipótese nula leva à conclusão da existência de efeitos individuais e, por conseguinte, à inadequação do modelo pooled. O teste de Hausman é um teste do tipo wald, em que a hipótese nula é a de que as diferenças nos coeficientes são sistemáticas, ou seja, tais coeficientes são ortogonais; condição essencial para o modelo de efeitos aleatórios. A rejeição da hipótese nula do teste de Hausman indica que a melhor escolha é o modelo de efeitos fixos (GREENE, 2008).

\section{O modelo teórico}

No presente estudo, as variáveis do modelo serão transformadas em logaritmo natural, com o objetivo de obter as elasticidades, e principalmente, para aproximar os valores das variáveis, pois as unidades de medidas são em dimensões diferentes, evitando possíveis problemas com a normalidade, heterocedasticidade e 
autocorrelação nos resíduos que possa existir. O modelo de dados em painel pode ser expresso da seguinte maneira:

$$
\ln C O_{2}=\beta_{0}+\beta_{1} \ln P I B+\beta_{2}(\ln P I B)^{2}+\varepsilon
$$

Onde:

$\mathrm{CO}_{2}$ é o dióxido de carbono;

PIB representa o PIB per capita;

$\varepsilon$ é o termo de erro estocástico.

A partir da utilização do modelo de dados em painel, os resultados do modelo serão gerados com o apoio do Software Gretl.

\section{RESULTADOS}

Para melhor explorar as características dos dados é importante realizar a estatística descritiva. A Figura 3 apresenta os Box-plots do $\mathrm{CO}_{2}$ para cada estado da região Norte.

Figura 3 - Box-plot do $\mathrm{CO}_{2}$ distribuído entre os estados.

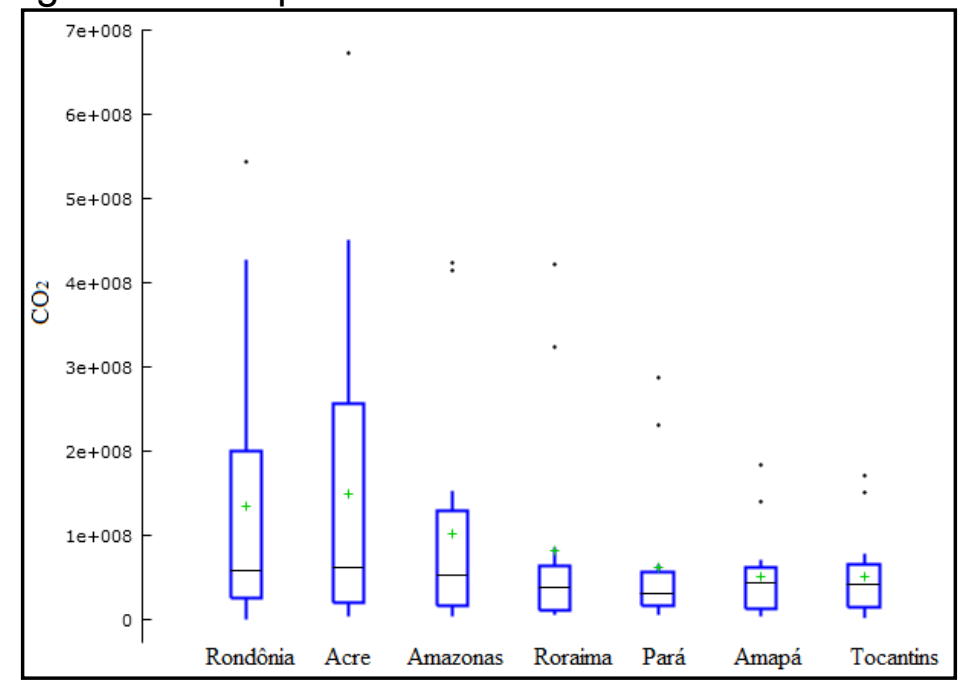

Fonte: Elaborada pelos autores.

Nessa figura, pode-se notar que Rondônia e Acre possuem as maiores variabilidades de emissão de $\mathrm{CO}_{2}$ nos anos estudados e que Roraima, Pará, Amapá e Tocantins mantiveram uma variabilidade pequena entre os anos.

A Figura 4 mostra quantidade de $\mathrm{CO}_{2}$ ao longo dos anos para cada estado. Esses resultados reforçam a visualização da figura anterior sobre os que mais emitem $\mathrm{CO}_{2}$ e os menos. É importante elucidar os picos de emissão nos anos de 2006 e 2013, o primeiro devido aos altos índices de desmatamento observados nessa região que, posteriormente, diminuiu (SEEG, 2018), enquanto o segundo é por causa também do crescimento do desmatamento observado naquele ano (INPE, 2018). 
Figura 4 - Série temporal do $\mathrm{CO}_{2}$ por estado nos anos de 2002 a 2015.

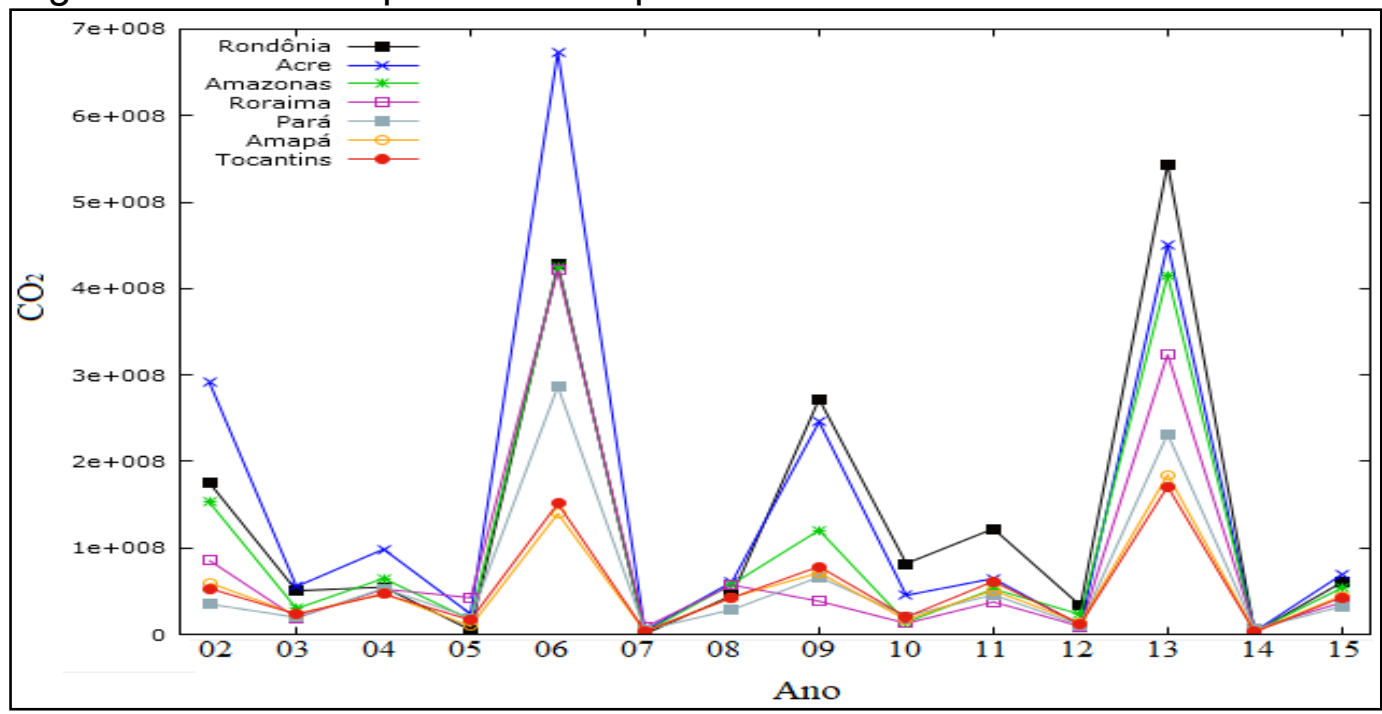

Fonte: Elaborada pelos autores.

A análise do PIB per capita se inicia pela Figura 5 que é composta pelos Boxplots para cada estado. É notável o crescimento do PIB per capita da esquerda para a direita entre os estados, com Rondônia e Acre possuindo os menores valores, enquanto que Tocantins, Amapá e Pará lideram com maiores valores.

Figura 5 - Box-plot do PIB per capita distribuído entre os estados.

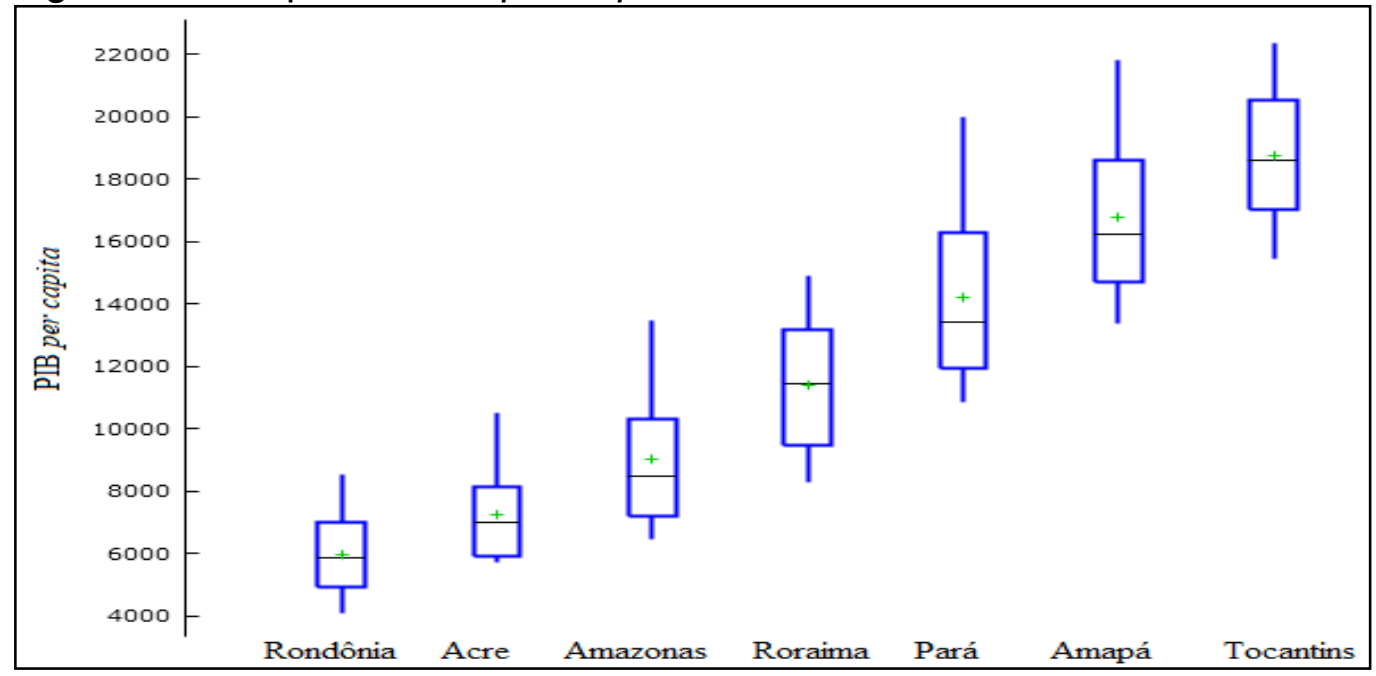

Fonte: Elaborada pelos autores.

Com apoio dos Box-plots feitos tanto para $\mathrm{O}_{\mathrm{CO}_{2}}$ quanto PIB per capita, observa-se que os estados que possuem altas emissões de $\mathrm{CO}_{2}$ tem baixo PIB per capita, indicando existir uma relação inversa entre eles.

Pode-se ser vista na Figura 6 a série temporal do PIB per capita por estado nos anos da análise. Como visto na figura anterior, o ranking dos estados com maiores e menores PIB per capita se mantêm ao longo dos anos, não existindo grandes diferenças de um ano para o outro, apresentando um comportamento aparentemente estacionário. 
Figura 6 - Série temporal do PIB per capita por estado nos anos de 2002 a 2015.

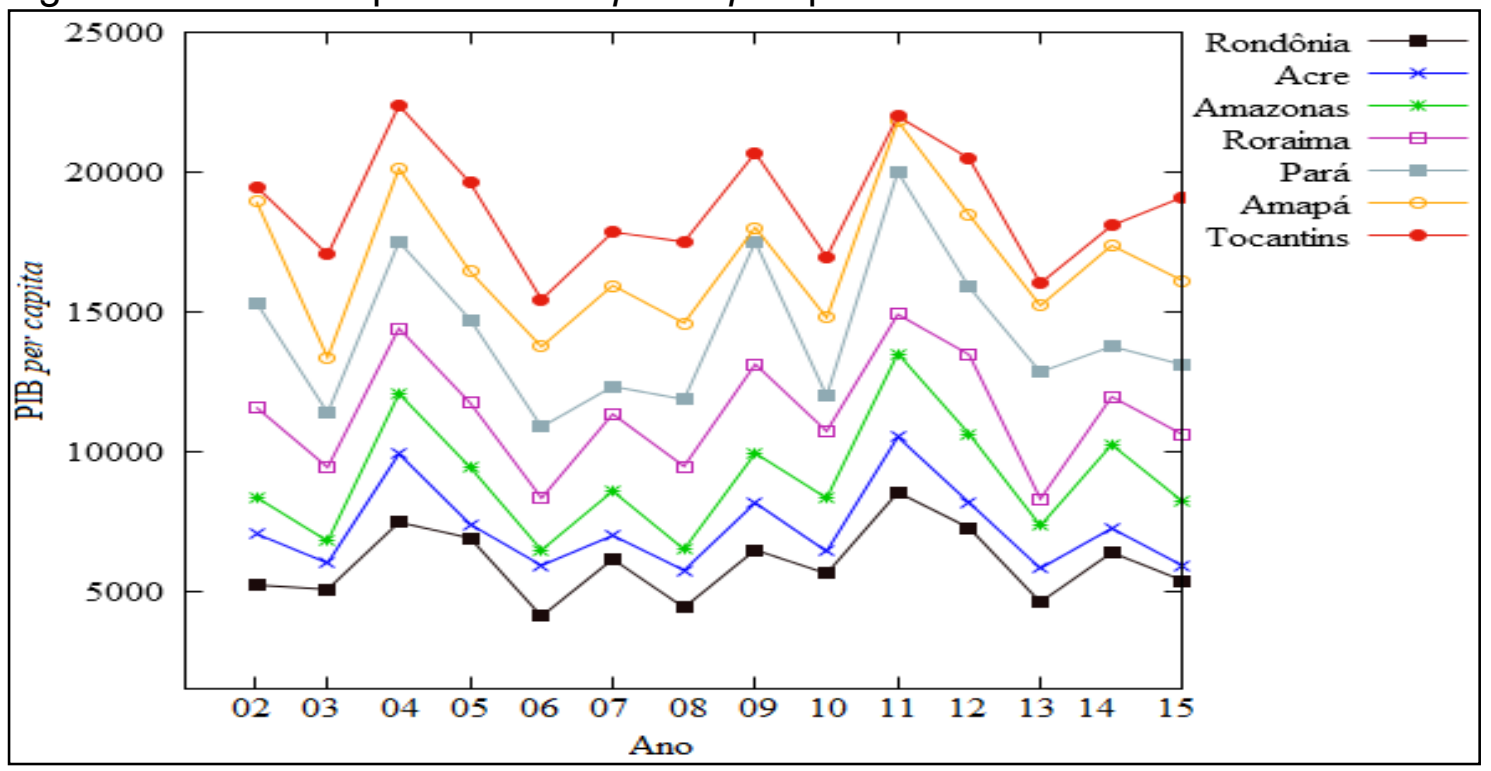

Fonte: Elaborada pelos autores.

Observa-se uma grande diferença entre os PIB per capita dos estados Rondônia e Acre para os estados Pará, Amapá e Tocantins. Isso se explica devido ao fato de o PIB daqueles estados não serem tão altos e a sua população estimada ser, relativamente, grande. Já nestes últimos estados, o PIB é alto e a população é mais adequada às proporções do PIB. O que permite que estes estados tenham uma renda per capita mais elevada.

\section{Aplicação do modelo de regressão para dados em painel}

Para aplicação do modelo de dados em painel adequado, utilizaram-se os testes de Chow, Hausman e Breusch-Pagan. A Tabela 1 mostra os resultados dos testes, incluindo as hipóteses para cada teste, seguido de seu $p$-valor e a decisão adequada.

Tabela 1 - Testes para escolha do modelo em painel.

\begin{tabular}{llcc}
\hline Testes & Hipóteses & p-valor & Decisão \\
\hline Chow & $\mathrm{H}_{0}:$ Pooled & 0,62 & Pooled \\
& $\mathrm{H}_{1}$ : Efeito fixo & & \\
\hline Hausman & $\begin{array}{l}\mathrm{H}_{0}: \text { Efeito aleatório } \\
\mathrm{H}_{1}: \text { Efeito fixo }\end{array}$ & 0,11 Efeito Aleatório \\
\hline $\begin{array}{l}\text { Breusch- } \\
\text { Pagan }\end{array}$ & $\begin{array}{l}\mathrm{H}_{0}: \text { Pooled } \\
\mathrm{H}_{1}: \text { Efeito aleatório }\end{array}$ & 0,19 Pooled \\
\hline
\end{tabular}

Fonte: Elaborada pelos autores .

Os resultados dos testes indicam que não há evidências para se rejeitar a hipótese nula em nenhuma deles, chegando a uma predominância pela escolha do modelo pooled, pois no teste Chow ao comparar o modelo pooled e efeito fixo a decisão da escolha é pelo pooled. O teste Hausman indica que o efeito aleatório é preferível ao de efeito fixo, e por último, o teste de Breusch-Pagan seleciona o modelo pooled ao de efeito aleatório. 
Após se realizar a escolha do modelo adequado, foram testadas as principais hipóteses da suposição do modelo, como pode ser visto na Tabela 2 a estatística de Durbi-Watson apresentou indícios de uma moderada autocorrelação negativa entre os resíduos, porém optou-se em não realizar sua correção para não influenciar na forma da curva, assim como sugerem Gujarati e Porter (2011) de que uma das alternativas é manter os resultados na presença de autocorrelação.

Tabela 2 - Testes ajustes para o modelo.

\begin{tabular}{lrr}
\hline \multicolumn{1}{c}{ Testes } & Estatística & p-valor \\
\hline Durbin - Watson & 2,96 & $0,10<$ \\
Normalidade & 5,00 & 0,08 \\
White & 10,07 & $0,04^{*}$ \\
\hline $\mathrm{R}^{2}$ & $6,20 \%$ & - \\
\hline \multicolumn{2}{c}{${ }^{*}$ Significativo a 5\%. }
\end{tabular}

Fonte: Elaborada pelos autores.

O p-valor associado ao teste de normalidade é maior que $5 \%$ de significância, portanto não existem evidências para se rejeitar a hipótese nula de normalidade nos resíduos. O baixo valor explicativo do modelo com $6,20 \%$ é decorrente ao fato das emissões de $\mathrm{CO}_{2}$ serem explicadas por diversos fatores que não estão inclusos no modelo para se representar a Curva de Kuznets Ambiental.

O teste de White verifica a homocedasticidade dos resíduos, o p-valor de 0,04 sugere que com nível de significância de $5 \%$ que os resíduos são heterocedásticos, apesar possuir um valor quase no limite da decisão.

O resultado da regressão pooled para dados em painel é vista na Tabela 3, essa é composta dos coeficientes estimados, seguida do erro padrão e p-valor associado a cada estimativa. A estimação do erro padrão para os coeficientes estimados foi por meio de erros padrão robustos para amenizar a autocorrelação e pequena heterocedasticidade.

Inicialmente, foi testado no modelo um termo cúbico, não sendo significativo por isso não foram mostrados seus resultados e já se realizou a regressão apenas com o termo quadrático, os resultados mostram que logaritmo natural do PIB per capita e seu termo ao quadrado foram significativos a $1 \%$, com o primeiro sendo negativo e o segundo positivo.

Tabela 3 - Resultado da regressão para o modelo de efeitos fixos.

\begin{tabular}{lrrrr}
\hline & COEFICIENTE & ERRO PADRÃO & RAZÃO-T & P-VALOR \\
\hline Const & 128,23 & 16,24 & 7,90 & $<0,01$ \\
Ln(PIB per capita) & $-23,27$ & 3,52 & $-6,60$ & $<0,01$ \\
${\text { [Ln(PIB per capita) }{ }^{2}}^{2}$ & 1,22 & 0,19 & 6,36 & $<0,01$ \\
\hline
\end{tabular}

Fonte: Elaborada pelo autor.

O modelo pode ser escrito como

$\operatorname{lnCO_{2}}=128,23-23,27 \times \ln P I B p c+1,22 \times(\ln P I B p c)^{2}$

Para o valor do PIB per capita que otimiza a emissão de $\mathrm{CO}_{2}$, deve-se como condição necessária derivar a função em relação ao PIB per capita e igualar a zero:

$$
\begin{gathered}
\frac{d \ln C O_{2}}{d \ln P I B p c}=-23,27 \times \frac{1}{P I B p c}+2 \times 1,22 \times \ln P I B p c \times \frac{1}{P I B p c}= \\
=\frac{-23,27+2,44 \ln P I B p c}{P I B p c}=0
\end{gathered}
$$

Efetuando operações básicas se chega ao seguinte resultado: 


$$
\begin{gathered}
\ln P I B p c=\frac{23,27}{2,44}=9,5369 \\
e^{\ln P I B p c}=e^{9,5369} \\
P I B p c=R \$ 13.861,91
\end{gathered}
$$

Utilizando a condição suficiente de segunda ordem, será preciso derivar novamente em relação ao PIB per capita, portanto será utilizada a regra do quociente, chegando ao seguinte resultado:

$$
\begin{aligned}
& \frac{d \ln C \mathrm{O}_{2}}{d \ln P I B p c}=\frac{-23,27+2,44 \ln P I B p c}{P I B p c} \\
& \frac{d^{2} \ln C \mathrm{O}_{2}}{d^{2} \ln P I B p c}=\frac{(-23,27+2,44 \ln P I B p c)^{\prime} \times P I B-(-23,27+2,44 \ln P I B p c) \times P I B p c^{\prime}}{P I B p c^{2}} \\
& \frac{d^{2} \ln C O_{2}}{d^{2} \ln P I B p c}=\frac{2,44 \times \frac{1}{P I B p c} \times P I B p c-(-23,27+2,44 \ln P I B p c) \times 1}{P I B p c^{2}} \\
& \frac{d^{2} \ln C O_{2}}{d^{2} \ln P I B p c}=\frac{25,71+2,44 \ln P I B p c}{P I B p c^{2}}>0
\end{aligned}
$$

Como tanto o numerador quanto denominador são positivos, temos pela condição suficiente que um PIB per capita de $\mathrm{R} \$ 13.861,91$ minimiza as emissões de $\mathrm{CO}_{2}$. Note que quando o PIB per capita é menor que o ponto mínimo, provoca uma diminuição nas emissões de $\mathrm{CO}_{2}$, porém ao se ultrapassar esse mínimo com acréscimos no PIB per capita provocam um aumento as emissões de $\mathrm{CO}_{2}$. Nesse sentido, o PIB per capita encontrado é um alerta de que mudanças no conjunto de variáveis que levam ao crescimento do PIB devem ser acompanhadas de melhorias na qualidade de vida ambiental.

De acordo com o modelo em sua forma quadrática, a Curva de Kuznets Ambiental é obtida na Figura 7. Percebe-se que a curva possui a forma de $U$, não sendo invertido como a teoria afirma, da mesma forma como encontrado no trabalho de Queiroz, Rocha e Queiroz (2014). Segundo os autores, o nível de substâncias alcançou em seus primeiros anos um nível considerado menor, no entanto, quando o PIB começa a apresentar um crescimento, teve também um aumento no nível de emissão das SDO, ocasionando uma maior deterioração do meio ambiente.

Figura 7 - Curva de Kuznets Ambiental entre os anos 2002 a 2015.

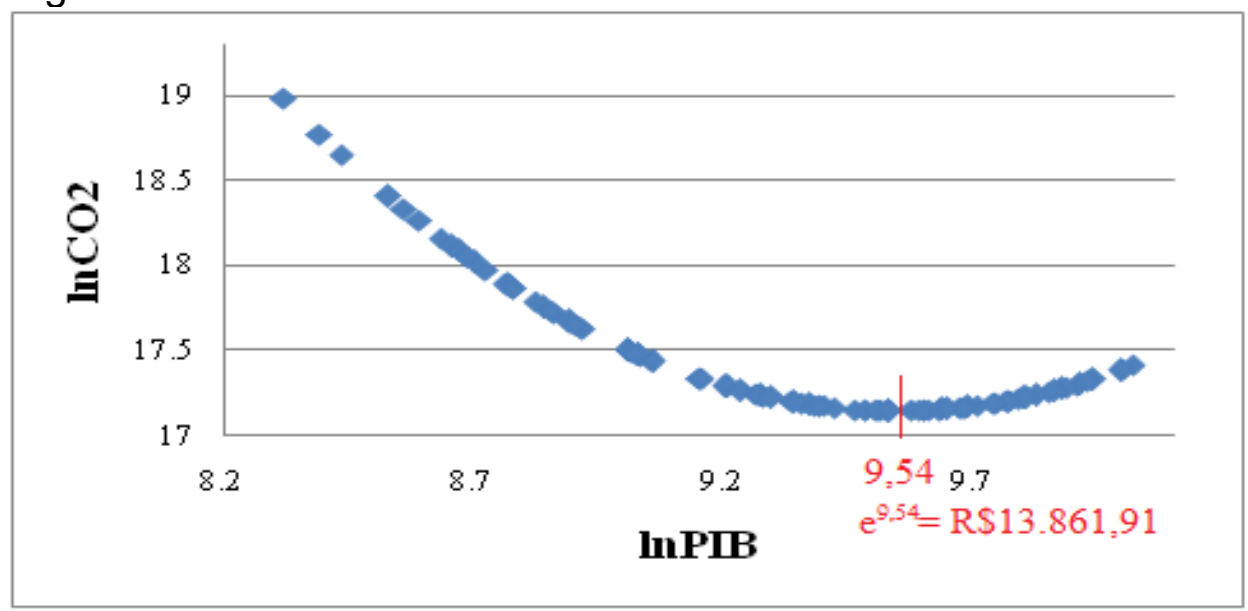

Fonte: Elaborada pelos autores. 
Além disso, o formato encontrado pode ser o início de uma curva no formato de $\mathrm{N}$ invertido, como encontrado na maioria dos trabalhos revisados no presente estudo. Os autores encontraram uma curva no formato de $\mathrm{N}$ invertido, que representa um movimento cíclico, tanto a nível nacional, que é o caso de Lucena (2005) e Oliveira et al, (2011), como a nível global, que é onde chegaram Arraes, Diniz \& Diniz (2006), Carvalho (2008), Carvalho (2010) e Catalán (2014). Esse formato indica que a degradação ambiental diminui inicialmente com o aumento do PIB, mas que volta a crescer depois, não proporcionando a qualidade de vida ambiental que a teoria propõe.

\section{CONSIDERAÇÕES FINAIS}

Este estudo teve como objetivo modelar uma Curva de Kuznets Ambiental para a região Norte do Brasil no período 2002 a 2015, a fim de confirmar que a CKA para a região é no formato de $U$ invertido. Neste sentido, foram utilizados dados colhidos no SEEG e SIDRA-IBGE, para, por meio da metodologia de dados em painel, realizar o exame da relação existente entre o PIB per capita e a emissão de $\mathrm{CO}_{2}$. Além, claro, de questionar-se o fato de que devido a região possuir especificidades, a curva modelada para a região seria na forma que a teoria expõe.

A partir da aplicação do modelo de regressão pooled, a fim de ajustar a CKA para a região Norte, verifica-se um baixo valor explicativo do modelo, o que se justifica devido ao fato das emissões de $\mathrm{CO}_{2}$ serem explicadas por diversos fatores (queima de combustíveis fósseis, desmatamentos, queimadas, etc.) e que alguns deles não estão inclusos no modelo para se representar a curva. Além disso, através do modelo proposto é possível chegar a constatação que um PIB per capita de $\mathrm{R} \$ 13.861,91$ minimiza as emissões de $\mathrm{CO}_{2}$, isto é, quando os estados possuírem valores menores que esse indica que as emissões de dióxido de carbono decaem, porém ao ultrapassálo provoca um aumento no $\mathrm{CO}_{2}$. Este resultado serve de alerta, pois com valores maiores que $R \$ 13.861,91$ existem indícios que os estados estão crescendo de forma a degradar o meio ambiente, emitindo cada vez mais dióxido de carbono. A partir desse ponto que se devem gerar políticas para incentivar o crescimento econômico por meio de tecnologias limpas, que possam proporcionar o crescimento contínuo, diminuindo ou mantendo-se constante a poluição por $\mathrm{CO}_{2}$.

Com a estimação da CKA para a região Norte, pode-se perceber que ela possui o formato de $U$ não invertido, sendo assim diferente do proposto pela teoria. Além disso, não se pode desconsiderar que a curva encontrada também possa adquirir o formato de $\mathrm{N}$ invertido. Ademais, quando se atinge elevados níveis de renda, a diminuição das emissões é cessada, possivelmente porque as oportunidades para reduzir as emissões estão diminuindo, os custos de redução tendem a aumentar porque o preço das novas tecnologias é maior.

Dessa maneira, pode-se perceber que o crescimento econômico por si só não gera uma melhora na qualidade de vida ambiental, é necessário que paralelamente a esse crescimento haja também um compromisso dos estados com a redução da emissão de $\mathrm{CO}_{2}$, ou seja, as políticas possuem um papel chave nesse processo. Não é somente em períodos de crescimento econômico que os acordos ambientais possam ter efetividade, é necessário considerar a capacidade institucional dos governos para alcançarem reduções relevantes na degradação do meio ambiente. aDiante do exposto sobre a relação crescimento econômico e a degradação do meio ambiente, sugere-se que novos trabalhos sejam feitos ampliando a série temporal para se verificar as tendências da curva e que novas proxies sejam adotadas para 
degradação ambiental e crescimento econômico, que represente de maneira melhor essas variáveis, inserindo outros fatores que sejam responsáveis pela curva.

\section{REFERÊNCIAS}

ARRAES, R. A.; DINIZ, M. B.; DINIZ, M. J. T. Curva ambiental de Kuznets e desenvolvimento econômico sustentável. RER, Rio de Janeiro, vol. 44, nº3, p. 525-547, jul/set 2006.

ÁVILA, E. S. de; DINIZ, E. M. Evidências sobre curva ambiental de Kuznets e convergência das emissões. Estud. Econ., São Paulo, vol.45, n.1, p. 97-126, jan.-mar. 2015.

BARROS, L.; GOMES, F.. Desigualdade e desenvolvimento: A hipótese de Kuznets é válida para os municípios brasileiros?. Revista Análise Econômica, v.50, p.57-81, 2008.

BALTAGI, B. H. Econometric analysis of panel data. Nova York: John Wiley and Sons, 1995.

BRASIL. Lei Complementar $n^{\circ}$ 124, de 3 de janeiro de 2007. Texto digitalizado. Disponível em: <http://www2.camara.gov.br/internet/legislacao/legin.html/ visualizarNorma.html?ideNorma=548988>. Acesso em 25 de fevereiro de 2018.

MMA. Conferência das Partes. Disponível em: <http://mma.gov.br/clima/convencao-das-nacoes-unidas/conferencia-das-partes>. Acesso em 03 de julho de 2018.

. Presidência da República. Plano Amazônia Sustentável: diretrizes para o desenvolvimento sustentável da Amazônia Brasileira. Brasília, 2008.

CALDARELLI, C. E.; CAMARA, M. R. G.; PERDIGÂO, C. Instituições de ensino superior e desenvolvimento econômico: o caso das universidades estaduais paranaenses. Planejamento e políticaspúblicas, n. 44, p.85-112, jan./jun. 2015.

CAMERON, A. C.; TRIVEDI, P. K. Microeconometrics: methods and applications. New York: Cambridge University Press, 2005.

CARVALHO, T. S. A hipótese da Curva de Kuznets Ambiental global e o Protocolo de Quioto. UFJF, Juiz de Fora, 2008.

CARVALHO, T. S.; ALMEIDA, E. A Hipótese da Curva de Kuznets Ambiental Global:Uma Perspectiva Econométrico-Espacial. Est. Econ., São Paulo, v. 40, n. 3, P. 587-615, jul-set, 2010.

CATALÁN, H.. Curva ambiental de Kuznetz: implicaciones para um crecimiento sustentable. Economía Informa, núm. 389, noviembre-diciembre, México, 2014.

GREENE, W. H. Econometric analysis.4. ed. Englewood Cliffs, NJ.: Prentice Hall, 2000 .

GROSSMAN, G. M.; KRUEGER, A. B. Environmental impacts of a North American free trade agreement. National Bureau of Economic Research, 1991. 
Economic growth and the environment. Quarterly Journal of Economics, v. 110, p. 353-377, 1995.

GROSSMAN, V..Inequality, Economic Growth, and Technological Change - New Aspects in an Old Debate. Heidelberg: New York: Physical-Verl, 2001.

IKEDA, D. Respeito universal pela dignidade humana: o grande caminho da paz. Proposta de Paz enviada às Nações Unidas (ONU), Japão, 2016.

INPE. INPE estima $5.843 \mathrm{~km}^{2}$ desmatados na Amazônia em 2013. Disponível em: $<$ http://www.INPE.br/noticias/noticia.php?Cod_Noticia=3443> . Acesso em 22 de fevereiro de 2018.

INSTITUTO BRASILEIRO DE GEOGRAFIA E ESTATÍSTICA - IBGE. Estimativas de População. Disponível em: <https://www.ibge.gov.br/estatisticasnovoportal/sociais/populacao/9103-estimativas-de-populacao.html?\&t=resultados>. Acesso em: 13 de fevereiro de 2018.

KUZNETS, Simon Smith. Economic growth and income inequality. American Economic Review, vol. 45, n.1, p. 1-28, 1955.

LOUREIRO, A.; COSTA, L.. Uma breve discussão sobre os modelos com dados em painel. Fortaleza: Instituto de Pesquisa e Estratégia Económica do Ceará (IPECE), 2009.

LUCENA, A. F. P. de. Estimativa de uma Curva de Kuznets Ambiental Aplicada ao Uso de Energia e suas Implicações para as Emissões de Carbono no Brasil. COPPE/UFRJ, Rio de Janeiro, 2005.

OliveiRA, R. C.; ALMEIDA, E. S.; FREGUGLIA, R.; BARRETO, R. C. S. Desmatamento e crescimento econômico no Brasil: uma análise da curva de Kuznets ambiental para a Amazônia legal. Revista de Economia e Sociologia Rural, v. 49, $n^{\circ}$ 3, p. 709-740, 2011.

QUEIROZ, Indiane Azevedo; ROCHA, Thiago; QUEIROZ, Índia Azevedo. Crescimento econômico com desenvolvimento sustentável: uma análise acerca da curva ambiental de Kuznets no Brasil entre 2002 a 2010. UNIMONTES, Minas Gerais, 2014.

ROCHA, L. A.; KHAN, A. S.; LIMA, P. V. P. S. Nível tecnológico e emissão de poluentes: uma análise empírica a partir da Curva de Kuznets Ambiental. Economia Aplicada, v. 17, n. 1, pp. 21-47, 2013.

REINALDO, L. M. ESTIMAÇÃO CLÁSSICA E BAYESIANA PARA DADOS EM PAINEL. UNB, Brasília, 2017.

SALVATO, M. A.; ALVARENGA, P. S.; FRANÇA, C. S.; ARAÚJO, A. F. de JR. Crescimento e desigualdade: evidências da Curva de Kuznets para os municípios de Minas Gerais 1991/2000. Revista Economia \& Gestão da PUC Minas, Minas Gerais, 2006.

SEEG. Mudança de Uso da Terra. Disponível em: <http://seeg.eco.br/mudanca-deuso-da-terra/>. Acesso em 25 de fevereiro de 2018. 
Emissões

Totais.

Disponível

em:

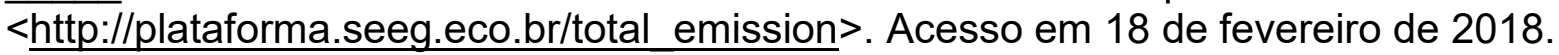
2017.

Nota Metodológica Setor Mudança de Uso do Solo e Florestas. Outubro,

SERRANO, A. L. M.; LOUREIRO, P. R. A.; NOGUEIRA, J. M. Evidência da Curva de Kuznets Ambiental no Brasil: uma análise do crescimento econômico e poluição. Revista Economia e Desenvolvimento, v. 13, n. 2, p. 304-314, 2014

SISTEMA IBGE DE RECUPERAÇÃO AUTOMÁTICA - SIDRA. Produto Interno Bruto dos Municípios. Disponível em: <https://sidra.ibge.gov.br/tabela/5938>. Acesso em: 13 de fevereiro de 2018.

WOOLDRIDGE, J. M. Econometric Analysis of Cross Section and Panel Data. The MIT Press, Cambridge, 2002.

WORLD WIDE FUND FOR NATURE - WWF.Missão Global. Disponível em: <http://www.natureza-portugal.org/wwf2/>. Acesso em: 20 de fevereiro de 2018. 